
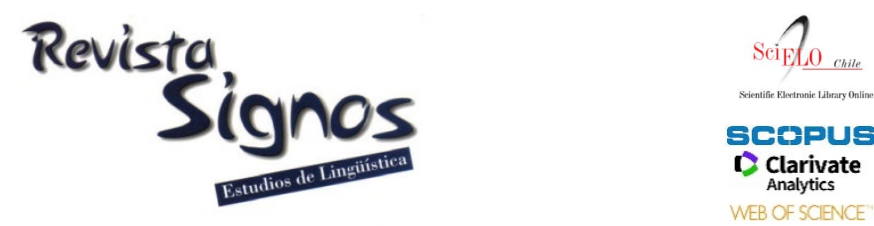

\title{
Revisitando la corporeidad del lenguaje narrativo
}

\author{
Revisiting the embodiment of narrative language \\ Manuel de Vega \\ UNIVERSIDAD DE LA LAGUNA \\ ESPAÑA \\ mdevega@ull.edu.es
}

Recibido: 12-VII-2021 / Aceptado: 05-XI-2021

DOI: $10.4067 /$ S0718-09342021000300985

\section{Resumen}

El enfoque corpóreo del significado postula que la comprensión de las palabras, oraciones o del discurso (especialmente el narrativo) reutiliza los sistemas neuronales de la percepción, la acción y la emoción. Los datos más recientes de la neurociencia apoyan esta idea. En particular, la neuroimagen y la electrofisiología cerebral confirman que el lenguaje referido a acciones induce activaciones en la corteza motora y premotora. No obstante, los críticos a la corporeidad consideran que estos datos no son concluyentes, ya que son meramente correlacionales y no demuestran que haya vínculos causales entre resonancia motora y significado lingüístico (v.g., Mahon \& Caramazza, 2008; Mahon, 2015; Dove, 2016; Ostarek \& Huettig, 2019). Es más, sugieren que el significado se procesa en un gran centro semántico de propósito general y que las activaciones sensoriomotoras no tendrían valor funcional alguno. Este artículo ofrece nuevas evidencias que apoyan fuertemente la idea de causalidad; es decir, las activaciones motoras serían parte substancial del significado. En primer lugar, los pacientes de Parkinson no solo tienen alterada su conducta motora, sino que muestran dificultades específicas en el uso de verbos de acción. En segundo lugar, cuando se pide a los participantes que lean textos, con las manos tras la espalda su recuerdo de oraciones de acción empeora. En tercer lugar, la estimulación no invasiva excitatoria de la corteza motora mejora la memoria del lenguaje de acción. Finalmente, el artículo discute brevemente las ventajas funcionales del significado corpóreo sobre el significado puramente simbólico, y también trata del lenguaje abstracto, uno de los mayores desafíos a las teorías corpóreas.

Palabras Clave: Lenguaje de acción, neuroimagen, ritmos cerebrales, estimulación cerebral no-invasiva, lenguaje abstracto. 


\begin{abstract}
The embodied approach to meaning posits that the comprehension of words, sentences and discourse (especially narrative) reuses the neural systems of perception, action and emotion. The most recent data of neuroscience support this idea. Particularly, neuroimaging and brain electrophysiology confirm that action-related language involves activations of motor and premotor cortex. However, critics of embodiment consider these data inconclusive, since they are merely correlational and do not demonstrate causal links between motor resonance and linguistic meaning (v.g., Mahon \& Caramazza, 2008; Mahon, 2015; Dove, 2016; Ostarek \& Huettig, 2019). Furthermore, they suggest that meaning is processed in a general-purpose semantic hub, and sensory-motor activations would not play any functional role. This article offers strong new evidence of causality; that is, motor activations would be substantial part of meaning. First, Parkinson patients not only have impaired motor behavior, but also show selective difficulties in the use of action verbs. Secondly, when participants read texts, keeping their hands behind the back, their recall of action sentences is impaired. Thirdly, applying excitatory non-invasive brain stimulation over the motor cortex improves memory for action language. Finally, the article briefly discusses the functional advantages of embodied meaning, and also addresses abstract language, one of the challenges of the embodied approach.
\end{abstract}

Key Words: Action language, neuroimaging, brain rhythms, non-invasive brain stimulation, abstract language.

\title{
INTRODUCCIÓN
}

En 2005, tuve el honor de visitar al profesor Giovanni Parodi en la Universidad de Valparaíso, quien me invitó a impartir un seminario en el excelente programa de doctorado que él dirigía. En ese mismo año, y en el mismo número de la Revista Signos. Estudios de Lingüistica se publicaron dos artículos, uno del propio Giovanni Parodi (Parodi, 2005) y otro mío (de Vega, 2005), que trataban sobre dos de las grandes áreas en el estudio de la comprensión del discurso: los textos expositivos y los textos narrativos, respectivamente. El importante artículo de Parodi, titulado La comprensión del discurso especializado escrito en ámbitos técnico-profesionales, se centraba en la comprensión y aprendizaje de textos expositivos muy especializados, y enfatizaba los aspectos estructurales del texto, mientras que mi artículo, titulado Lenguaje, corporeidad y cerebro: Una revisión crítica trataba de las aportaciones de la perspectiva corpórea del significado poniendo el énfasis en la comprensión del discurso narrativo. Ambos artículos reflejan dos campos de investigación del discurso diferentes en cuanto a objetivos y métodos, pero también complementarios. Por una parte, los textos expositivos son productos culturales dirigidos a la adquisición de conocimientos, que suelen utilizarse en entornos educativos o académicos y su lectura requiere, generalmente, gran esfuerzo cognitivo, dado que se refieren a información conceptual altamente especializada, muy alejada de la experiencia cotidiana de la gente. Frecuentemente, el interés de su estudio es no sólo conocer sus aspectos formales (sintaxis, estructura, etc.), sino mejorarlos para facilitar su comprensión y para que sirvan mejor como herramientas de aprendizaje. Por su parte, el estudio de los textos narrativos, aun siendo también productos culturales, nos acerca más a los mecanismos más básicos y universales del lenguaje. El lenguaje humano 
es principalmente narrativo; incluso en las sociedades más primitivas e iletradas, las personas se cuentan todo tipo de relatos episódicos, personales o mitológicos, y en la sociedad actual, además de seguir intercambiando relatos, consumimos masivamente información narrativa, ya sea leyendo novelas, a través del cine, o de la inmensa oferta de series de televisión.

El presente artículo es un breve en ensayo, que en cierto modo es una actualización del mencionado artículo sobre la corporeidad del lenguaje (de Vega, 2005). En los más de 15 años trascurridos desde entonces, la investigación sobre la corporeidad del lenguaje narrativo ha ido acumulando nuevos datos, aportados por técnicas de investigación cada vez más sofisticadas. Pero también han surgido nuevas dudas y debates sobre la naturaleza del significado que recogeré aquí.

\section{Nuevos datos favorables a la corporeidad}

La doctrina corpórea postula que el significado está íntimamente imbricado en los sistemas perceptivos, motores y emocionales del cerebro (Glenberg \& Kaschack, 2002; Fischer \& Zwaan, 2008; Barsalou, Santos, Kyle Simmons \& Wilson, 2008; García \& Ibáñez, 2018). Según el enfoque corpóreo, el significado de las palabras, de las frases y de la mayor parte del discurso narrativo consiste en la simulación mental de los objetos, eventos o situaciones a los que se refieren. Por ejemplo, si escuchamos o leemos la frase 'Pedro golpeó el clavo con el martillo', se activarían fugazmente en nuestra mente imágenes visuales, auditivas, e incluso motoras y emocionales de la escena. Y si explorásemos nuestra actividad cerebral deberíamos observar, en efecto, activaciones en la corteza visual, motora, o auditiva de modo análogo, aunque quizá con menos intensidad, que durante la realización de la acción correspondiente.

La dimensión motora del significado ha sido particularmente investigada, empleando diversos métodos. Así, la técnica de imagen de resonancia magnética funcional (fMRI) permite registrar los cambios en el flujo sanguíneo cerebral asociados a la actividad cognitiva. De este modo se ha comprobado que al comprender oraciones de acción (e.g., escribir la carta) se activan áreas del córtex motor y premotor, que en parte se solapan con las correspondientes a la ejecución u observación de dichas acciones (e.g., Tettamanti, Manenti, Della Rosa, Falini, Perani, Cappa \& Moro, 2008; Moody \& Gennari, 2010; Urrutia, Gennari \& de Vega, 2012; de Vega, León, Hernández, Valdés, Padrón \& Ferstl, 2014; Yang, Dickey, Fiez, Murphy, Mitchell, Collinger, Tyler-Kabara, Boninger \& Wang, 2017). Estas activaciones no ocurren durante la comprensión de oraciones abstractas (e.g., dudar de la solución).

Por ejemplo, en un estudio se registró la actividad cerebral mediante neuroimagen de fMRI durante la comprensión de oraciones referidas a acciones que implican diferente grado de esfuerzo (Urrutia et al., 2013). Por ejemplo, 
- Como Pedro decidió pintar el salón, está moviendo la foto (bajo esfuerzo)

- Como Pedro decidió pintar el salón, está moviendo el sofá (alto esfuerzo)

- Como Pedro decidió pintar el salón, está eligiendo un color (no esfuerzo)

Los resultados mostraron mayor activación en la corteza parietal inferior izquierda (PII) cuando los participantes leían frases de alto esfuerzo que cuando leían frases de bajo esfuerzo o de no esfuerzo (Figura 1A). La corteza PII también se activaba cuando los mismos participantes ejecutaban una acción manual (apretar una pelota). Esta región cerebral está relacionada con procesos de planificación de la acción, que incluyen la evaluación del tamaño, el peso y la secuencia de músculos que hay que movilizar para ejecutar la acción. De modo que es comprensible que el mayor esfuerzo, al requerir más planificación, active la corteza PII en mayor medida. Pero, no olvidemos que en este caso los participantes no realizaban ninguna acción, sino que únicamente comprendían descripciones de acciones.

Una característica bien conocida del cerebro es que continuamente produce ritmos electrofisiológicos de varios tipos. Se sabe por los estudios de electroencefalografía (EEG) que, en concreto, los llamados ritmos mu, de distribución fronto-central y frecuencia de 8-13 Hz, están altamente sincronizados en estado de reposo, pero se desincronizan cuando los individuos ejecutan una acción manual o cuando observan una acción manual de otra persona; por tanto, los ritmos $m u$ son marcadores neuronales de los procesos motores. Lo más interesante es que los ritmos mu también se desincronizan cuando las personas entienden oraciones con verbos referidos a acciones manuales. Por ejemplo, Moreno, de Vega y León (2013) presentaban a los participantes videos que mostraban acciones manuales y escuchaban frases referidas a acciones manuales (e.g., 'ahora corto el pan') y frases abstractas (e.g., 'ahora dudo del plan'), mientras se registraba su EEG. Los resultados mostraron una fuerte desincronización del ritmo $m u$ con distribución fronto-central tanto durante la observación de acciones como durante la escucha de frases de acción manual, pero no ante las frases abstractas, indicando una activación de los procesos motores (Figura 1A y Figura 1B). En un estudio posterior se corroboró esta modulación de mu por el lenguaje de acción y se obtuvo que la fuente cerebral de este efecto está el córtex premotor (Moreno, de Vega, León, Bastiaansen, Lewis \& Magyari, 2015). 


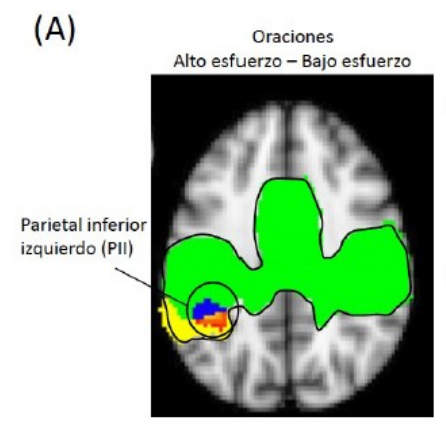

(B)

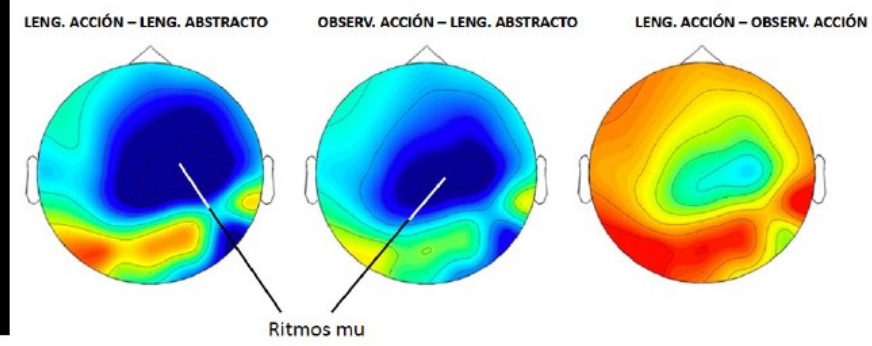

Figura 1A. Resultados de un estudio de neuroimagen (fMRI), que muestra activación diferencial entre oraciones de alto esfuerzo y de bajo esfuerzo en el lóbulo parietal inferior

izquierdo (PII); la amplia región bilateral en gris claro corresponde a la ejecución de movimientos reales de las manos (basado en Urrutia et al., 2013). Figura 1B. Resultados de un estudio de EEG, que muestran la distribución topográfica de las diferencias en modulación de ritmos mu. Comparado con el lenguaje abstracto, tanto el lenguaje de acción como la observación de videos de acción modulan los ritmos fronto-centrales mu (izquierda y centro, respectivamente), mientras que el contraste entre lenguaje de acción y observación

de acción (derecha) no produjo efectos diferenciales (basado en Moreno et al., 2013).

En conclusión, las evidencias favorables al significado corpóreo son múltiples. Tanto los datos de neuroimagen como electrofisiológicos confirman la implicación de los mecanismos motores del cerebro en la comprensión del lenguaje de acción. Estos datos se unen a los de otros estudios, que aquí no revisaremos, que muestran que la comprensión de oraciones referidas a movimientos visuales (e.g., el caballo saltó sobre la valla) activa aquellas áreas del córtex visual responsables de procesar el movimiento, o las oraciones de contenido emocional inducen la activación del cerebro emocional. No obstante, los datos revisados en este apartado son esencialmente correlacionales y no demuestran un vínculo causal entre activaciones cerebrales y comprensión del significado. Por otra parte, los estudios de neuroimagen ofrecen información detallada sobre anatomía funcional, pero carecen de resolución temporal, mientras que los datos de EEG tienen gran resolución temporal pero escasa resolución espacial.

\section{Críticas y alternativas a la corporeidad del significado}

En conjunto, los estudios anteriores coinciden en mostrar una activación de la corteza motora y premotora durante la comprensión del lenguaje de acción, respaldando la idea de que, en algunos casos, el significado lingüístico implica simulaciones motoras. Sin embargo, sigue habiendo escépticos (Mahon \& Caramazza 2008; Mahon 2015; Dove, 2016). Un argumento 'anti-corpóreo' habitual es que el significado no se puede identificar con la resonancia de la corteza motora, ya que las personas que nunca tocaron la guitarra o jamás montaron en bicicleta y, por lo tanto, no tienen esas habilidades en su repertorio motor, aún podrían entender frases como 'tocar la guitarra' y 'montar en bicicleta'. El caso sería aún más extremo en las personas que tienen alterados o 
paralizados sus procesos motores debido a alguna enfermedad y, en consecuencia, no deberían ser capaces de entender ninguna frase de acción. Una respuesta tentativa a estas afirmaciones es que las personas que carecen de determinadas habilidades motoras aún están expuestas a otras fuentes de información corpóreas (por ejemplo, imágenes visuales o auditivas de la acción de tocar la guitarra). Una respuesta más empírica sería demonstrar que, de hecho, la falta de habilidades motoras determina dificultades en la comprensión del lenguaje de acción, tal como veremos en un próximo apartado. Un segundo argumento crítico es que los datos experimentales esgrimidos en favor de la corporeidad son esencialmente correlacionales, y no demuestran necesariamente que la resonancia del cerebro motor tenga un impacto causal en el procesamiento del significado. Este es un razonamiento técnicamente correcto (correlación no implica causalidad) y por ello requiere aportar nuevas pruebas empíricas como veremos. Finalmente, un problema del enfoque corpóreo del significado se relaciona con el lenguaje abstracto; es decir, que la propuesta corpórea es razonable para explicar el lenguaje referido a contenidos sensorio-motores, pero no parece apropiada para explicar el significado de conceptos como 'verdad', 'dudar', etcétera.

Por otra parte, recordemos que una alternativa a las teorías corpóreas son las teorías simbolistas, que postulan que el significado es esencialmente conceptual y abstracto. Hasta hace poco, el planteamiento simbolista no contaba con gran apoyo en los datos neurocientíficos. Sin embargo, algunas propuestas recientes de la neurosemántica, aun reconociendo que en la comprensión del lenguaje se producen activaciones sensoriomotoras distribuidas en el cerebro, consideran que el significado lingüístico depende sobre todo de mecanismos semánticos de propósito general, que representan información puramente conceptual. En particular, hay evidencia de que el lóbulo temporal anterior (ATL), bilateralmente, es un centro semántico general (bub) donde se procesa el significado, en formato amodal, es decir, independientemente del contenido o de la modalidad sensorial de la información (Lambon Ralph, Pobric \& Jefferies, 2009). La relevancia del centro semántico está avalada por varios estudios. Así, los pacientes con demencia semántica sufren un proceso neurodegenerativo en el ATL bilateral y como consecuencia muestran un deterioro extremo en una variedad de tareas semánticas, como nombrar imágenes, relacionar palabras con imágenes, verificar si las palabras se refieren a imágenes, clasificar las palabras de acuerdo con su significado, clasificar los objetos de acuerdo con la similitud, demostrar el uso adecuado de los objetos o reconocer objetos en función de características visuales, auditivas, somatosensoriales o gustativas / olfativas (Lambon Ralph \& Patterson, 2008). Los estudios de neuroimagen funcional en participantes sanos también revelan una actividad bilateral significativa en el ATL cuando éstos realizan tareas léxico-conceptuales y la inhibición del ATL por medio de neuroestimulación reduce temporalmente el rendimiento en tales tareas (Binney \& Ralph, 2015). 
No se puede negar la relevancia funcional del centro semántico de propósito general, representado por los lóbulos temporales anteriores, en el procesamiento del significado. Pero ¿quiere esto decir que los procesos corpóreos observados son irrelevantes? La temporalidad de las activaciones corpóreas puede dar pistas, ya que un procesamiento temprano de estas representaciones podría apoyar la idea de funcionalidad (Ostarek \& Huettig, 2019). Sin embargo, los críticos de las teorías de la corporeidad afirman que las activaciones de las áreas sensorio-motoras son un mero epifenómeno sin consecuencias funcionales ya sea porque ocurren demasiado tarde (Mahon \& Caramazza, 2008; Mahon, 2015), o porque ocurren demasiado pronto en el curso del procesamiento léxico (Kintsch, 2008; Graesser \& Jackson, 2008). Así, para Mahon y Caramazza (2008) las activaciones corpóreas son procesos asociativos que ocurren tardíamente 'en cascada', después de haberse procesado el significado en el centro semántico (Mahon \& Caramazza, 2008). Por el contrario, desde la teoría de ‘construcción-integración' de Walter Kintsch se propone que las activaciones sensorio-motoras son muy tempranas $\mathrm{y}$ tan fugaces que se desvanecen antes de poder integrarse en los procesos del significado (Kintsch, 2008; Graesser \& Jackson, 2008). En cualquier caso, es algo extraño suponer que las activaciones sensorio-motoras, con el consiguiente gasto metabólico cerebral, sean funcionalmente inútiles. Las propuestas híbridas según las cuales el significado combina representaciones corpóreas y simbólicas son relevantes (Louwerse \& Jeuniaux, 2008). Por ejemplo, una opción perfectamente posible es que, al recibir las palabras, se activen inicialmente las regiones sensorio-motoras, que harían un primer procesamiento específico de contenidos (motores, visuales, emocionales, etc.) y luego se comunicarían con ATL, que funcionaría como gran centro de integración multimodal (García, Moguilner, Torquati, García-Marco, Herrera, Muñoz, Castillo, Kleneschay, Sedeño \& Ibáñez, 2019). Si esto fuera así, tendríamos una explicación híbrida del significado: las representaciones corpóreas tienen funciones especializadas de simulación, y el ATL de integración multimodal y ambos procesos son igualmente funcionales. Pero, además de estos argumentos es importante demostrar empíricamente que las activaciones corpóreas son causalmente necesarias para procesar el significado lingüístico, tal como veremos en el siguiente apartado.

\section{Pruebas de causalidad de las representaciones corpóreas}

Vamos a mencionar tres tipos de estudios que aportan pruebas sobre los vínculos causales entre los procesos corpóreos del cerebro y el significado lingüístico. Concretamente, mencionaremos los estudios con pacientes cerebrales, las investigaciones sobre el influjo de las representaciones corpóreas en la memoria y los estudios de estimulación cerebral no invasiva. Se ha comprobado que los pacientes con enfermedad de Parkinson, que tienen dañados los ganglios basales del sistema motor, no sólo muestran alteraciones motoras, sino que también tienen dificultades selectivas para procesar el lenguaje de acción. Por ejemplo, Herrera y Cuetos (2012) informaron que dichos pacientes antes de recibir el tratamiento dopaminérgico tienen 
dificultad selectiva para describir imágenes que representan acciones, aunque no muestran dificultad en nombrar objetos. Lo más interesante es que, en esos mismos pacientes, la dificultad en el uso de los verbos de acción se desvanece después de recibir la medicación, lo cual indica una clara relación causal entre procesos motores cerebrales y lenguaje de acción.

Algunos escépticos anti-corpóreos consideran que las activaciones sensorio-motoras son momentáneas y fugaces -una especie de fuegos de artificio- sin consecuencias cognitivas a largo plazo (Kintsch, 2008; Graesser \& Jackson, 2008). Recientemente se ha visto que no es así. En un experimento realizado por Dutrieux, Dahiez y Gyselinck (2019) los participantes recibían listas de frases que incluían un verbo de acción (v.g., 'colgar un bastón') o un verbo atencional (v.g., 'observar un bastón'). Durante la lectura de algunas listas debían mantener las manos delante apoyadas sobre la mesa, mientras que en otras listas mantenían las manos agarradas tras la espalda. Después de cada lista, y tras realizar una tarea distractiva, los participantes debían recordar las frases. Los resultados indicaron que la postura de manos detrás reducía el recuerdo de las oraciones de acción, pero no a las atencionales, indicando que dicha postura interfería con la simulación mental de la acción afectando a la memoria a largo plazo. Más recientemente, de Vega, Dutriaux, Moreno, García-Marco, Seigneuric y Gyselinck (2021) desarrollaron un experimento análogo registrándose al mismo tiempo la actividad cerebral mediante EEG. Los resultados conductuales fueron idénticos a los del estudio anterior: la postura manos detrás reducía el recuerdo de las frases de acción. Además, mantener las manos delante producía desincronización de ritmos 'beta' fronto-centrales -al igual que los ritmos $m u$, un marcador de actividad motora- mientras mantenerlas detrás eliminaba ese efecto. Es decir, que esta última postura interfería con los procesos motores en el cerebro, impidiendo la simulación de las acciones y causando una menor tasa de aciertos en una tarea posterior de memoria. Ambos estudios demuestran, en definitiva, una relación causal entre actividad del cerebro motor y la codificación y recuerdo de oraciones de acción.

En la misma línea, los protocolos de estimulación cerebral no invasiva son especialmente útiles, ya que pueden modificar la actividad de un área cerebral determinada para observar las consecuencias conductuales, estableciendo así una posible relación causal entre cerebro y conducta. Un modo de hacerlo es creando 'lesiones virtuales', es decir inhibiendo la zona de interés. Por ejemplo, Vukovic, Feurra, Shpektor, Myachykov y Shtyrov (2017) administraron estimulación magnética transcraneal repetitiva (rTMS) para reducir la actividad de la corteza motora primaria y hallaron que los juicios semánticos relativos a las palabras de acción se enlentecieron, mientras que no se alteraban los juicios sobre palabras abstractas. De nuevo, alterar el funcionamiento del cerebro motor afectó sólo al lenguaje de acción proporcionando evidencia causal de la corporeidad. 
El estudio de Vukovic et al. (2017) Muestra un claro vínculo causal o funcional entre activación motora y juicios semánticos. No obstante, el efecto observado fue inmediato y no es posible saber si persistía en el tiempo. Más recientemente, Vitale, Padrón, Avenanti y de Vega (2021) pidieron a los voluntarios que acudiesen a dos sesiones de laboratorio. En una de ellas recibían estimulación eléctrica transcraneal, es decir no invasiva, sobre la corteza motora primaria (M1), correspondiente a la mano derecha, durante 20 minutos. La mitad de los participantes recibían estimulación excitatoria (electrodo anódico sobre M1) y la otra mitad estimulación inhibitoria (electrodo catódico sobre M1). La otra sesión era de control y todos los voluntarios recibían 20 minutos de estimulación falsa sobre M1. En ambas sesiones, tras la estimulación real o falsa, los participantes recibían varios bloques de frases simples, referidas a acciones manuales o atencionales. Cada bloque de frases era seguido de una prueba de memoria guiada en que se presentaban sólo los verbos y debían recordar el objeto correspondiente. El resultado más importante fue que la estimulación anódica (excitatoria) de M1 mejoró el recuerdo de las frases de acción, pero no de las frases atencionales tal como se aprecia en la Figura 2.

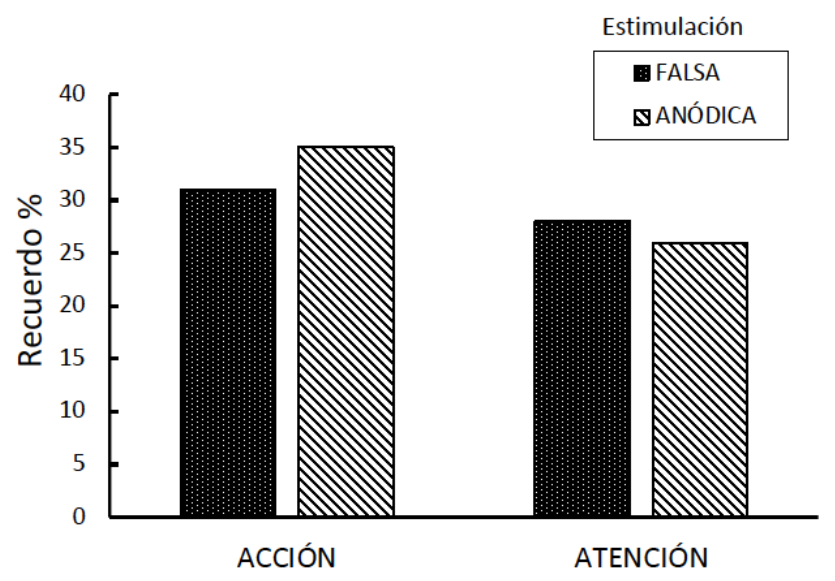

Figura 2. Porcentaje de recuerdo de las oraciones de acción y atencionales, tras la estimulación falsa o de control y la estimulación eléctrica anódica (excitatoria) sobre la corteza motora (basado en Vitale et al., 2021).

En suma, los estudios con pacientes de Parkinson, los estudios de memoria con manipulación de la postura corporal y el uso de estimulación cerebral no invasiva demuestran que la actividad de la corteza motora tiene una relación causal directa con el procesamiento del lenguaje de acción y, además, el impacto de las representaciones corpóreas no se limita al procesamiento inmediato, sino que tiene consecuencias sobre la memoria a largo plazo. 


\section{Ventajas de la corporeidad del significado}

Las evidencias empíricas favorables a la corporeidad del significado lingüístico que acabamos de revisar son abrumadoras. Pero ¿cómo podría ser de otro modo? Las ventajas de un sistema lingüístico 'conectado' directamente con el mundo mediante representaciones o simulaciones corpóreas son varias. Mencionemos algunas. El significado corpóreo facilita la interfaz con el mundo físico, facilita la comunicación entre interlocutores y proporciona una evaluación emocional inmediata de lo que leemos o escuchamos.

El significado corpóreo prepara para la acción, al proporcionar una interfaz más directa entre el lenguaje y el mundo físico que un sistema de representación simbólica. Muchos de los mensajes verbales que producimos y comprendemos se refieren al entorno físico, y esto obliga a los interlocutores a establecer el referente de las palabras en su experiencia perceptiva y motora inmediata. Por ejemplo, si alguien me dice "por favor, alcánzame la botella', para responder a esta petición tendré que localizar 'la botella' en el campo visual próximo, debo también localizar físicamente al hablante para poder identificar el referente del pronombre 'me' (en alcánzame), debo planificar una acción motora bastante compleja para materializar la acción referida con 'alcánzame'; esto es desplazar primero la mano y agarrar la botella, y luego trasladar la botella hasta las proximidades del hablante. Todo esto parece más factible si, al comprender la oración, construimos una simulación corpórea de toda esta secuencia a partir de las palabras. En cambio, no está muy claro cómo podría hacerse esto a partir de una representación puramente simbólica abstracta. Estas últimas, por su carácter abstracto, arbitrario y amodal tendría dificultades para acoplarse a nuestra experiencia del mundo; es decir, el famoso problema de la toma de tierra (grounding).

Una función primordial del lenguaje es la comunicación, y para que ésta tenga éxito es preciso que haya un buen ajuste o alineamiento entre las representaciones que construyen el hablante y el oyente (Garrod \& Pickering, 2004). Dicho de otro modo, las personas nos entendemos en la medida en que nos representamos los referentes lingüísticos de modo similar. Un modo eficiente de conseguir ese alineamiento de representaciones entre los interlocutores es construir simulaciones corpóreas, utilizando la maquinaria cerebral perceptivo-motora que todos compartimos.

El lenguaje es un eficaz vehículo de las emociones y, por tanto, el significado lingüístico implica una evaluación corpórea que va más allá de la pura semántica. Como asegura Damasio (1994), el cerebro emocional primario (básicamente el sistema límbico y la amígdala) evolucionó en los animales para asegurar la supervivencia, al generar respuestas corporales muy intensas (las emociones) ante estímulos de gran valor adaptativo, como los riesgos o amenazas físicas (por ejemplo, la presencia de un depredador). Pero cuando se desarrollan cerebros complejos como el nuestro, capaces de pensar o de utilizar el lenguaje, la información más 'cognitiva' también se evalúa 
corporalmente con la misma maquinaria emocional. Por ello el cerebro humano ha incorporado conexiones entre el sistema límbico y la corteza frontal, que garantizan que las experiencias más 'abstractas' sean evaluadas corporal y emocionalmente. Así, las palabras y las frases pueden producir una intensa reacción emocional en el que las escuchas; pensemos en las emociones que pueden suscitar, una mala noticia, una buena noticia, un insulto, o un halago. Los mismos mecanismos que velan por la supervivencia física, en contextos naturales de amenaza, nos proporcionan una aprehensión inmediata, a través de nuestro cuerpo, del valor biológico del significado lingüístico.

Un ejemplo empírico de la conexión emoción-lenguaje es un experimento de Havas, Glenberg, Gutowski, Lucarelli y Davidson (2010), que demostró que las expresiones faciales tienen un papel funcional en la comprensión de oraciones emocionales. En el estudio participaron mujeres que recibieron un tratamiento antiarrugas, consistente en la inyección subcutánea de la toxina botulínica en los músculos del entrecejo, que produce el efecto de paralizarlos. Se observó que después del tratamiento las participantes tuvieron más dificultad (mayor tiempo de lectura) en la comprensión de oraciones relativas a situaciones de ira (p. ej., 'harto de discutir con aquel cabezota intolerante, te fuiste dando un portazo') o de pena (p. ej., 'contuviste tus lágrimas al entrar en la sala del funeral'), mientras que su comprensión de situaciones alegres (p. ej., 'subes corriendo las escaleras del apartamento de tu amor') no se alteraba. Es decir, la parálisis de los músculos del entrecejo, que intervienen en la expresión de emociones negativas como enfado y pena, dificulta la comprensión de oraciones que implican dichas emociones, lo cual sugiere que la simulación de la expresión facial emocional es necesaria para la comprensión.

Las propuestas simbólicas, dado el problema de toma de tierra de los símbolos, tienen serias dificultades para afrontar las cuestiones que acabamos de mencionar. Pero ¿qué decir de otras propuestas como el conexionismo? Se trata éste de un enfoque que, en cierta medida, emula los mecanismos computacionales del cerebro. Se concibe como una inmensa red de nodos ampliamente distribuidos e interconectados, de modo que la fuerza de sus conexiones se altera (se fortalece o debilita) en función del entrenamiento con estímulos lingüísticos (letras, fonemas o palabras), mediante la aplicación de ciertas reglas de aprendizaje (v.g., Marcus, 2001). El simbolismo clásico también propone la existencia de una 'red semántica', pero hay una diferencia: en el simbolismo los nodos son símbolos discretos que corresponden a palabras o conceptos individuales, mientras que en el conexionismo los nodos son sub-simbólicos y -como las neuronas del cerebrocontribuyen a computar el significado, pero no constituyen en sí mismos unidades de significado. Dicho esto, el conexionismo adolece del mismo problema de toma de tierra que el simbolismo clásico: los nodos en la red están desvinculados del mundo sensoriomotor y por tanto carecen de referencia (de Vega, Graesser \& Glenberg, 2008). 


\section{El problema del significado abstracto}

La mayoría de los experimentos que apoyan la corporeidad han utilizado como material lingüístico palabras u oraciones concretas que se refieren a objetos, acciones o reacciones emocionales. Estos son contenidos importantes en la cognición y en el lenguaje humanos, pero las personas también usan el lenguaje para referirse a experiencias más allá de las proporcionadas por palabras y oraciones concretas. Pensemos en nombres como 'verdad', o 'justicia', verbos como 'recordar', o 'dudar' que no se refieren a objetos o eventos perceptivos. O bien, en los términos gramaticales como la negación 'no' con su inversión de valor de verdad (Kaup \& Zwaan, 2003), el concesivo 'a pesar de' con valor contra- argumentativo (Parodi, Julio, Nadal, Cruz \& Burdiles, 2019), o el pronombre 'esto' con su función encapsuladora del discurso (Parodi \& Burdiles, 2019), que aparentemente son sólo reguladores intralingüísticos. Asimismo, las metáforas se refieren a conceptos abstractos, pese a utilizar términos concretos. En general, la abstracción supone un desafío para el enfoque de la corporeidad, porque las palabras y oraciones abstractas a menudo no parecen estar basadas en experiencias corpóreas. Lo deseable sería demostrar que las palabras y oraciones abstractas también se basan en alguna medida en la percepción, la acción o la emoción.

En algunos casos, las teorías de la corporeidad proporcionan explicaciones apropiadas para el significado abstracto. Por ejemplo, el significado metafórico es abstracto, aunque podría activar representaciones corpóreas. Así, el tiempo puede expresarse en términos de espacio (Santiago, Lupiáñez, Pérez \& Funes, 2007; Casasanto \& Boroditsky, 2008) los eventos positivos y negativos pueden expresarse en términos de la dimensión vertical espacial (Meier \& Robinson, 2004; Santana \& de Vega, 2011), la exclusión social puede expresarse como dolor físico (Eisenberger, Lieberman \& Williams, 2003), la indignación moral como asco (Moll, Oliveira-Souza, Garrido, Bramati, Caparelli-Daquer, Paiva, Zahn \& Gennari, 2007), etc. Las investigaciones neurocientíficas, en efecto muestran que la actividad cerebral es compartida por el significado metafórico y la correspondiente experiencia corpórea. Pero las metáforas son un caso especial de significado híbrido porque, aunque se refieren a ideas abstractas, su soporte lingüístico sigue siendo concreto (Lakoff \& Johnson, 1980).

Con relación a los marcadores gramaticales, pensemos en la negación, un operador lingüístico francamente abstracto que existe en todos los idiomas. Sin embargo, hay razones para pensar que incluso la negación tiene en su origen un carácter corpóreo. Los datos evolutivos muestran que, en la primera infancia, cuando los niños sólo utilizan expresiones de una o dos palabras, ya entienden y usan negaciones en contextos de acciones concretas, para expresar 'rechazo' (e.g., ante la pregunta de la madre '¿quieres yogurt?’ el niño responde ‘NNo, no!') o 'prohibición' (e.g., la madre va a coger el osito y el niño dice '¡No!, mío'). Es decir, que hay una estrecha asociación temprana entre negación e inhibición de acciones (Pea, 1982; Austin, Theakston, Lieven \& Tomasello, 
2014). En una etapa posterior del desarrollo, los niños frecuentemente se hablan a sí mismos empleando negaciones como una forma de auto-prohibiciones, que indican una internalización de la negación. Solo más tarde, la negación adquiere un carácter declarativo para expresar ideas más abstractas no ligadas a acciones (e.g., 'no acepto tu explicación', 'no tiene voluntad'). Por otra parte, los estudios con adultos muestran que la comprensión de oraciones negativas reduce la activación de algunas áreas cerebrales y hace menos accesibles en la memoria los conceptos negados, lo cual sugiere procesos de inhibición semántica (MacDonald \& Just, 1989). Esto permite plantear la hipótesis siguiente: los mecanismos responsables de la inhibición de respuestas podrían estar involucrados en el procesamiento de frases negativas (de Vega, Morera, León, Beltrán, Casado \& Martín-Loeches, 2016). La inhibición de respuesta consiste en cancelar acciones planificadas o suprimir comportamientos alternativos o representaciones que sean inapropiadas, inseguras o ya no sean necesarias. En la vida diaria continuamente suprimimos acciones físicas o mentales sobre la marcha, como cuando detenemos el coche ante el semáforo en rojo, rectificamos una decisión que habíamos tomado o desechamos un pensamiento molesto. En el laboratorio se pueden inducir procesos inhibitorios mediante el paradigma Go-NoGo, en el que en la mayoría de los ensayos los participantes deben apretar una tecla ante una señal Go (ej., un círculo azul) y refrenar su respuesta en el caso infrecuente de recibir una señal NoGo (ej., círculo amarillo). Si se registra el EEG, se observa que los ensayos NoGo aumentan la potencia de los ritmos cerebrales theta, que son característicos de los procesos de inhibición motora ejecutados en el lóbulo prefrontal. En una serie de estudios de EEG (de Vega et al., 2016; Beltrán, Morera, García-Marco \& de Vega, 2019; Liu, Wang, Beltrán, Gu, Liang, Wang \& de Vega, 2020), los participantes leyeron frases afirmativas (e.g., 'Ahora sí cortarás el pan') y negativas (e.g., 'Ahora no cortarás el pan'), simultáneamente a la realización (Go) o inhibición (NoGo) de una respuesta motora. Los resultados indicaron que la negación modulaba los ritmos theta en los ensayos NoGo, indicando que la comprensión de negaciones y la inhibición motora comparten mecanismos neurales. El mecanismo inhibitorio parece operar no solo en las negaciones referidas a acciones, sino también durante la comprensión de negaciones puramente declarativas como 'Ahora no deseas una sorpresa', que modulan los mismos marcadores de la inhibición como los ritmos theta del EEG (Beltrán et al., 2019).

Pero ¿qué decir de palabras como 'verdad' o 'justicia', que no tienen claros referentes en el mundo físico? Algunos investigadores creen que, incluso en estos casos, la comprensión implica una simulación corpórea de situaciones concretas. Por ejemplo, Barsalou y Wiemer-Hastings (2005) reflexionan sobre la comprensión del concepto abstracto 'verdad', y aseguran que, si bien la palabra presentada de forma aislada no tiene ningún referente sensorial, lo habitual es que aparezca en el contexto de frase y pragmático que le proporciona un andamiaje situacional. Por ejemplo: 'María ha dicho que está lloviendo y es "verdad". En el contexto de esta frase el lector puede simular 
mentalmente la situación como una serie de operaciones perceptivo-motoras en las que el concepto 'verdad' resulta mucho más concreto. Es decir, se representaría sucesivamente: a) un personaje diciendo algo sobre un estado del mundo (está lloviendo); b) un oyente que construye una representación de lo que dice el hablante (la expectativa de lluvia); c) el oyente explora visualmente la parte del mundo a la que se refiere la afirmación del hablante (cómo está el tiempo ahí fuera); d) y verifica si el significado de la frase original se ajusta al estado del mundo que acaba de verificar; e) si se da un ajuste entre significado y estado del mundo, el hablante concluye que es VERDAD, en caso contrario, que es FALSO.

No obstante, el tema del significado abstracto no queda totalmente resuelto por las teorías corpóreas del significado. Muchos conceptos lingüísticos que utilizamos constantemente no se corresponden a estados o eventos físicos del mundo, sino más bien a estados internos, fruto de la introspección del hablante, como ilustran los adjetivos 'aburrido' o 'feliz', o los verbos: 'creer', 'desear', o 'dudar'. En estos casos, más que hablar de un significado estrictamente corpóreo puede ser útil postular procesos más propios de la llamada 'teoría de la mente'; es decir, de los mecanismos neuronales que nos permiten entender las intenciones y creencias de uno mismo y de los demás. Una idea relevante es que las palabras abstractas funcionan como 'herramientas sociales', que se adquieren en contextos de interacción humana, y que al usarlas modifican en gran medida el entorno y las relaciones sociales con otras personas (Borghi, Barca, Binkofski, Castelfranchi, Pezzulo \& Tummolini, 2019; Borghi, 2020). Por ejemplo, supongamos un contexto real en que un amigo nos dice: 'la 'justicia' me ha condenado'. Los términos abstractos ‘justicia' y 'condenado' cobrarían un significado social (cómo cambiará mi relación con mi amigo), propioceptivo (qué emociones siento) y hasta sensorio-motoras (imágenes de la sala de justicia y de los jueces) muy claras. Según Borghi, desde un punto de vista cerebral, los términos abstractos implicarían redes neurales relacionados con la cognición social y con los movimientos de la boca, ya que las palabras abstractas se utilizan principalmente en la comunicación oral entre personas.

\section{CONCLUSIONES}

La acumulación de evidencia científica sobre la realidad del significado corpóreo, especialmente la procedente de la neurociencia del lenguaje, es incontestable. Los estudios de neuroimagen, de electrofisiología cerebral, de pacientes con trastornos neurodegenerativos o de estimulación no invasiva ofrecen datos convergentes sobre el carácter sensorio-motor del significado. Además, se ha confirmado que las representaciones corpóreas forman parte sustancial de significado (tienen valor causal), y no son meros epifenómenos. No obstante, hay desafíos para el enfoque corpóreo que deben afrontarse para consolidar las teorías. En primer lugar, la base neuronal del significado está ampliamente distribuida y podría tener una organización funcional jerárquica. El significado no sólo implicaría activaciones corpóreas, sino también 
mecanismos de integración multimodal, como el centro ATL. Por ello, será necesario investigar la división del trabajo entre las distintas redes neuronales del significado y la conectividad funcional entre ellas. En segundo lugar, para explicar el lenguaje abstracto se requiere seguramente extender y modificar la idea de corporeidad a los estados internos (propioceptivos, emocionales, cognitivos) y a las relaciones interpersonales, es decir, más allá de la percepción y acción sobre objetos. En tercer lugar, los postulados de corporeidad se han desarrollado principalmente en relación con el lenguaje narrativo, y es preciso afrontar el discurso expositivo esencialmente conceptual, impersonal y abstracto.

\section{REFERENCIAS BIBLIOGRÁFICAS}

Austin, K., Theakston, A., Lieven, E. \& Tomasello, M. (2014). Young children's understanding of denial. Developmental psychology, 50(8), 2061-2070.

Barsalou, L. \& Wiemer-Hastings, K. (2005). Situating abstract concepts. En D. Pecher \& R. Zwaan (Eds.), Grounding cognition: The role of perception and action in memory, language, and thought (pp. 129-163). Nueva York: Cambridge University Press.

Barsalou, L. W., Santos, A., Kyle Simmons, W. \& Wilson, C. D. (2008). Language and simulation in conceptual processing. En M. de Vega, A. Glenberg \& A. Graesser (Eds.), Symbols and embodiment: Debates on meaning and cognition (pp. 245284). Oxford: Oxford University Press.

Beltrán D., Morera, Y., García-Marco, E. \& de Vega, M. (2019). Brain inhibitory mechanisms are involved in the processing of sentential negation, regardless of its content. Evidence from EEG theta and beta rhythms. Frontiers in Psychology. DOI: 10.3389 /fpsyg.2019.01782

Binney, R. J. \& Ralph, M. A. L. (2015). Using a combination of fMRI and anterior temporal lobe rTMS to measure intrinsic and induced activation changes across the semantic cognition network. Neuropsychologia, 76, 170-181. DOI: 10.1016/j.neuropsychologia.2014.11.009

Borghi, A. M. (2020). A future of words: Language and the challenge of abstract concepts. Journal of Cognition, 3(1), 42.

Borghi, A. M., Barca, L., Binkofski, F., Castelfranchi, C., Pezzulo, G. \& Tummolini, L. (2019). Words as social tools: Language, sociality and inner grounding in abstract concepts. Physics of life reviews, 29, 120-153.

Boulenger, V., Mechtouff, L., Thobois, S., Broussolle, E., Jeannerod, M. \& Nazir, T. A. (2008). Word processing in Parkinson's disease is impaired for action verbs but not for concrete nouns. Neuropsychologia, 46(2), 743-756. DOI: 10.1016/j.neuropsychologia.2007.10.007 
Casasanto, D. \& Boroditsky, L. (2008). Time in the mind: Using space to think about time. Cognition, 106, 579-593. DOI: 10.1016/j.cognition.2007.03.004

Damasio, A. R. (1994). Descartes' error: Emotion, rationality and the human brain. Nueva York: Putnam.

de Vega, M. (2005). Lenguaje, corporeidad y cerebro: Una revisión crítica. Revista Signos. Estudios de Lingüistica, 38(58), 157-176.

de Vega, M., Graesser, A. \& Glenberg, A. (2008). Reflecting on the debate. En M. de Vega, A. Glenberg \& A. Graesser (Eds.), Symbols and embodiment: Debates on meaning and cognition. Oxford: Oxford University Press. DOI: 10.1093/acprof:oso/9780199217274.001.0001

de Vega, M., León, I., Hernández, J. A., Valdés, M., Padrón, I. \& Ferstl, E. (2014). Action Sentences Activate Sensory Motor Regions in the Brain Independent of Their Status of Reality. Journal of Cognitive Neuroscience, 26, 1363-1376.

de Vega, M., Morera, Y., León, I., Beltrán, D., Casado, P. \& Martín-Loeches, M. (2016). Sentential Negation Might Share Neurophysiological Mechanisms with Action Inhibition. Evidence from Frontal Theta Rhythm. The Journal of Neuroscience, 36, 602-610.

de Vega, M., Dutriaux, L., Moreno, I. Z., García-Marco, E., Seigneuric, A. \& Gyselinck, V. (2021). Crossing hands behind your back reduces recall of manual action sentences and alters brain dynamics. Cortex, 140, 51-65.

Dove, G. (2016). Three symbol ungrounding problems: Abstract concepts and the future of embodied cognition. Psychonomic Bulletin \& Review, 23(4), 1109e1121. DOI: $10.3758 /$ s13423-015-0825-4

Dutriaux, L., Dahiez, X. \& Gyselinck, V. (2019). How to change your memory of an object with a posture and a verb. Quarterly Journal of Experimental Psychology, 72(5), 1112e1118. DOI: $10.1177 / 1747021818785096$

Eisenberger, N. I., Lieberman, M. D. \& Williams, K. D. (2003). Does rejection hurt? An fMRI study of social exclusion. Science, 302(5643), 290-292.

Fischer, M. H. \& Zwaan, R. A. (2008). Embodied language: A review of the role of themotor system in language comprehension. Q J Exp Psychol, 61, 825-850.

García, A. M. \& Ibáñez, A. (2018). When embodiment breaks down: Language deficits as novel avenues into movement disorders. Cortex, 100, 1-7. 
García, A. M., Moguilner, S., Torquati, K., García-Marco, E., Herrera, E., Muñoz, E., Castillo, E., Kleneschay, T., Sedeño, L. \& Ibáñez, A. (2019). How meaning unfolds in neural time: Embodied reactivations can precede multimodal semantic effects during language processing. NeuroImage, 197, 439-449.

Garrod, S. \& Pickering, M. J. (2004). Why is conversation so easy? Trends in cognitive sciences, 8(1), 8-11.

Glenberg, A. \& Kaschak, M. P. (2002). Grounding language in action. Psychon Bull Rev. $9,558-565$.

Graesser, A. \& Jackson, G. T. (2008). Body and symbol in Auto'Tutor: Conversations that are responsive to the learners' cognitive and emotional states. En M. de Vega, A. Glenberg \& A. Graesser (Eds.), Symbols and embodiment: Debates on meaning and cognition (pp. 245-284). Oxford: Oxford University Press.

Havas, D. A., Glenberg, A., Gutowski, K. A., Lucarelli, M. J. \& Davidson, R. J. (2010). Cosmetic use of botulinum toxin-A affects processing of emotional language. Psychological Science, 21, 895-900.

Herrera, E. \& Cuetos, F. (2012). Action naming in Parkin'on's disease patients on/off dopamine. Neuroscience letters, 513(2), 219-222. DOI: 10.1016/j.neulet.2012.02.045

Kaup, B. \& Zwaan, R. (2003). Effects of negation and situational presence on the accessibility of text information. Journal of Experimental Psychology: Learning, Memory, and Cognition, 29, 439-446.

Kintsch, W. (2008). Symbol systems and perceptual representations. En M. de Vega, A. Glenberg \& A. Graesser (Eds.), Symbols and embodiment: Debates on meaning and cognition (pp.145-164). Oxford: Oxford University Press.

Lakoff, G. \& Johnson, M. (1980). Metaphors we live by. Chicago: University of Chicago Press.

Lambon Ralph, M. A. \& Patterson, K. (2008). Generalization and differentiation in semantic memory: Insights from semantic dementia. Annals of the New York Academy of Sciences, 1124(1), 61-76.

Lambon Ralph, M. A., Pobric, G. \& Jefferies, E. (2009). Conceptual knowledge is underpinned by the temporal pole bilaterally: Convergent evidence from rTMS. Cerebral Cortex, 19(4), 832-838. 
Liu, B., Wang, H., Beltrán, D., Gu, B., Liang, T., Wang, X. \& de Vega, M. (2020). The generalizability ofinhibition-related processes in the comprehension of linguistic negation. ERP evidence from the Mandarin language. Language, Cognition and Neuroscience, 35(7), 885-895.

Louwerse. M. \& Jeuniaux, P. (2008). Language comprehension is both embodied an symbolic. En M. de Vega, A. Glenberg \& A. Graesser (Eds.), Symbols and Embodiment. Debates on Meaning and Cognition (pp. 309-326). Nueva York: Oxford University Press.

MacDonald, M. C. \& Just, M. A. (1989). Changes in activation levels with negation. Journal of Experimental Psychology: Learning, Memory, and Cognition, 15(4), 633-642.

Mahon, B. Z. (2015). What is embodied about cognition? Lang Cogn Neurosi, 30, 420429.

Mahon, B. Z. \& Caramazza, A. (2008). A critical look at the embodied cognition hypothesis and a new proposal for grounding conceptual content. Journal of Physiology - Paris, 102, 59-70.

Marcus, G. F. (2001). The algebraic mind: Reflections on connectionism and cognitive science. Cambridge, MA: MIT Press.

Meier, B. P. \& Robinson, M. D. (2004). Why the sunny side is up: Associations between affect and vertical position. Psychol. Sci, 15, 243-247.

Moll, J., Oliveira-Souza, R. D., Garrido, G. J., Bramati, I. E., Caparelli-Daquer, E. M., Paiva, M. L., Zahn, R. \& Grafman, J. (2007). The self as a moral agent: Linking the neural bases of social agency and moral sensitivity. Social neuroscience, 2(3-4), 336-352.

Moody, C. L. \& Gennari, S. P. (2010). Effects of implied physical effort in sensorymotor and pre-frontal cortex during language comprehension. Neuroimage, 49(1), 782-793.

Moreno, I., de Vega, M. \& León, I. (2013). Understanding action language modulates oscillatory mu and beta rhythms in the same way as observing actions. Brain and cognition, 82(3), 236-242.

Moreno, I., de Vega, M., León, I., Bastiaansen, M., Lewis, A. G. \& Magyari, L. (2015). Brain dynamics in the comprehension of action-related language. A timefrequency analysis of mu rhythms. Neuroimage, 109, 50-62.

Ostarek, M. \& Huettig, F. (2019). Six challenges for embodiment research. Current Directions in Psychological Science, 28(6), 593-599. 
Parodi, G. (2005). La comprensión del discurso especializado escrito en ámbitos técnico-profesionales: ¿Aprendiendo a partir del texto? Revista Signos. Estudios de Lingüistica, 38(58), 221-267.

Parodi, G. \& Burdiles, G. (2019). Los pronombres neutros 'esto', 'eso' y 'aquello' como mecanismos encapsuladores en el discurso de la economía: Coherencia referencial y relacional. Spanish in Context, 16(1), 104-127.

Parodi, G., Julio, C., Nadal, L., Cruz, A. \& Burdiles, G. (2019). Stepping back to look ahead: Neuter encapsulation and referent extension in counter-argumentative and causal relations in Spanish. Language and Cognition, 11(3), 431-454.

Pea, R. D. (1982). Origins of verbal logic: Spontaneous denials by two-and three-year olds. Journal of child language, 9(3), 597-626.

Santana, E. \& De Vega, M. (2011). Metaphors are embodied, and so are their literal counterparts. Frontiers in Psychology, 2, 90.

Santiago, J., Lupiáñez, J., Pérez, E. \& Funes, M. J. (2007). Time (also) flies from left to right. Psychon. Bull. Rev, 14, 512-516. DOI:10.3758/BF03194099

Tettamanti, M., Manenti, R., Della Rosa, P. A., Falini, A., Perani, D., Cappa, S. F. \& Moro, A. (2008). Negation in the brain: Modulating action representations. Neuroimage, 43(2), 358-367.

Urrutia, M., Gennari, S. P. \& de Vega, M. (2012). Counterfactuals in action: An fMRI study of counterfactual sentences describing physical effort. Neuropsychologia, 50(14), 3663-3672.

Vitale, F., Padrón, I., Avenanti, A. \& de Vega, M. (2021). Enhancing motor brain activity improves memory for action language: A tDCS study. Cerebral Cortex, 31(3), 1569-1581.

Vukovic, N., Feurra, M., Shpektor, A., Myachykov, A. \& Shtyrov, Y. (2017). Primary motor cortex functionally contributes to language comprehension: An online rTMS study. Neuropsychologia, 96, 222-229.

Yang, Y., Dickey, M. W., Fiez, J., Murphy, B., Mitchell, T., Collinger, J., Tyler-Kabara, E., Boninger, M. \& Wang, W. (2017). Sensorimotor experience and verbcategory mapping in human sensory, motor and parietal neurons. Cortex, 92, 304-319. 